\title{
Development of High Intensity D-T fusion NEutron Generator (HINEG)
}

\author{
Yican Wu*, Chao Liu, Gang Song, Yongfeng Wang, Taosheng Li, Jieqiong Jiang, Yong Song, Xiang Ji, FDS Team \\ Key Laboratory of Neutronics and Radiation Safety, Institute of Nuclear Energy Safety Technology, Chinese Academy of Sciences, \\ Hefei, Anhui, 230031, China \\ *Corresponding Author, Email: yican.wu@fds.org.cn
}

\begin{abstract}
A high intensity D-T fusion neutron generator (HINEG) is keenly needed for the research and development (R\&D) of nuclear technology and safety of the advanced nuclear energy system, especially for the radiation protection and shielding. The R\&D of HINEG includes two phases: HINEG-I and HINEG-II. HINEG-I is designed to have both the steady beam and pulsed beam. The neutron yield of the steady beam is up to $10^{12}$ $\mathrm{n} / \mathrm{s}$. The width of pulse neutron beam is less than $1.5 \mathrm{~ns}$. HINEG-I is used for the basic neutronics study, such as measurement of nuclear data, validation of neutronics methods and software, validation of radiation protection and so on. HINEG-II aims to generate a high neutron yield of $10^{13} \mathrm{n} / \mathrm{s}$ neutrons by adopting high speed rotating tritium target system integrated with jet/spray array enhanced cooling techniques, and can further upgrade to obtain neutron yield of $10^{14} \sim 10^{15} \mathrm{n} / \mathrm{s}$ by using of accelerators-array in a later stage. HINEG-II can be used for fundamentals research of nuclear technology including mechanism of materials radiation damage and neutronics performance of components, radiation shielding as well as other nuclear technology applications.
\end{abstract}

\section{KEYWORDS: Fusion, Accelerator Based Neutrons Source, High Intesity, Radiation Shielding}

\section{INTRODUCTION}

Fusion energy becomes essential to solve the energy problem with the increase of energy demands. Although the recent studies of fusion energy have demonstrated the feasibility of fusion power, it commonly realizes that more hard work is needed on neutronics and safety before real application of fusion energy, especially on radiation protection and radiation biological effect of fusion neutron ${ }^{[1]}$. However the intensity of D-T neutron generators currently on operation around the world is lower than $10^{13} \mathrm{n} / \mathrm{s}$, which is severely restricting the research capability.

The Institute of Nuclear Energy Safety Technology (INEST), Chinese Academy of Sciences (CAS)/ has launched the High Intensity fusion Neutron Generator (HINEG) project to develop an accelerator-based D-T fusion neutron generator with the neutron yield higher than $10^{14 \sim 15} \mathrm{n} / \mathrm{s}$. HINEG project is divided into two stages. At the first stage, a D-T fusion neutron generator with both steady and pulse neutrons (HINEG-I) will be developed. The intensity will reach $10^{12} \mathrm{n} / \mathrm{s}$. At the second stage, a high intensity D-T fusion neutron generator will be developed. The intensity will reach $10^{14-15} \mathrm{n} / \mathrm{s}$. In this paper, the design and progress of HINEG will be described.

\section{DEVELOPMENT OF HIGH INTENSITY D-T FUSION NEUTRON SOURCES}

HINEG-I has two beam lines. The intensity of the steady beam could reach $10^{12} \mathrm{n} / \mathrm{s}$. For the pulse beam, the full width at half maximum (FWHM) is smaller than 1.5 ns. HINEG-I can be used for the basic research for fusion neutron science. HINEG-I can also be utilized in nuclear technology applications, such as neutron radiography, neutron activation analysis, neutron radiation detection and so on. At present, all components of HINEG-I have been developed. The installation has been finished (see Figure1 and Figure 2) and is commissioning in December, 2015.

HINEG-I will focus on the basic research of neutronics, including nuclear data measurement, verification and validation $(\mathrm{V} \& \mathrm{~V})$ of the methods and code, etc. For example, it can be used for the measurement of the (n, $p),(n, \alpha)$ cross sections of transmutation reactions that produce gases and affect the service performance of structure materials in fusion reactors, and the $(n, \gamma)$ reaction which contributes to the nuclear heating deposition in superconducting coils [2-4]. It is also a significant platform to perform the $\mathrm{V} \& \mathrm{~V}$ for the neutronics simulation software, such as SuperMC, and its applicability to fusion neutron environment [5]. Note that the SuperMC code was developed by INEST/FDS Team and has been validated by 2000 benchmarking cases for both fission and fusion reactors.

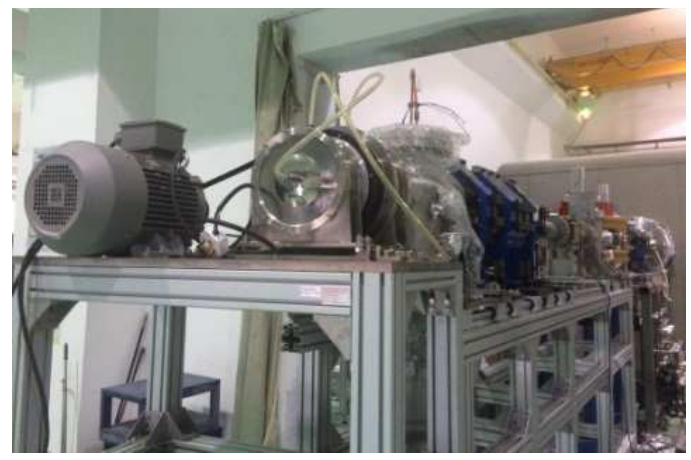

Figure 1: Steady beam line 


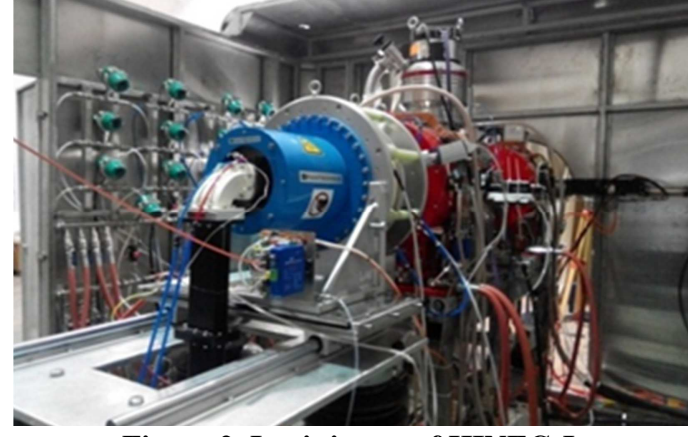

Figure 2. Ion injector of HINEG-I.

During the second phase (HINEG-II), in order to reach a neutron yield higher than $10^{14} \sim 10^{15} \mathrm{n} / \mathrm{s}$, several key technologies have been developed, such as the spraying impingement cooling for the target as well as a high intensity ion source. The feasibility of technological route is demonstrated. Furthermore, the neutron yield of $10^{15} \sim 10^{16} \mathrm{n} / \mathrm{s}$ is expected to be achieved through adopting accelerator-array and upgrading the rotating target. The design and development of key components of HINEG-II is ongoing.

HINEG-II will focus on fundamental phenomena of nuclear technology, including mechanism of material irradiation damage and neutronics performances of key components. For example, a series of material irradiation experiments will be performed to study irradiation effects on the mechanical properties of the China Low Activity Martensitic steel (CLAM), the primary candidate structural material for CN ITER TBM(Test Blanket Module), in high energy and high intensity neutron environment [6]. A series of experiments for fusion TBM, such as the Dual Function Lithium Lead liquid TBM and Helium Cooled Ceramic breeder TBM, will be performed to confirm whether the neutronics performance, such as tritium breeding ratio, nuclear heating deposition and material activation behavior, is consistent with the design or not.

\section{RESEARCH AND DEVELOPMENT OF KEY TECHNOLOGIES}

In order to produce high current ion beams, an optional concept is proposed, the accelerator is equipped with a ion source extracted a high intensity beam, which is installed (with its associated LEBT) inside a HV cabinet and connected to a $\mathrm{HV}$ accelerator column. At present, we have started to study the development difficulties and feasibility analysis of the whole accelerator.

During the transportation of HINEG-II, various of beam instabilities occurred due to space charge effect, which is partially compensated by the interaction of the beam with the residual gas. Such space charge compensation can be calculated to allow simulating beam trajectories. During the operation of HINEG-II, the power of $\mathrm{D}^{+}$ion beams hitting on the tritium target can be higher than $50 \sim 100 \mathrm{~kW} / \mathrm{cm}^{2}$. The temperature must be controlled bellow $200{ }^{\circ} \mathrm{C}$ in order to prevent the diffusion of tritium. To enhance the heat transfer capacity of tritium target system, jet array, spraying impingement, and nano-fluid cooling enhancement technologies are explored. A high heating target experiment platform have been developed (see Figure 3), and the technology verified experimental results show that the temperature of tritium target can be control under $180{ }^{\circ} \mathrm{C}$, while the beam current on the tritium target is bigger than 500 $\mathrm{mA}$. The comparison of the experimental and the theoretical results (see Figure 4) indicated that the heat transfer capability can reached to $57 \mathrm{~kW} /\left(\mathrm{m}^{2} \cdot \mathrm{K}\right)$, which has verified the feasibility of the cooling technologies.

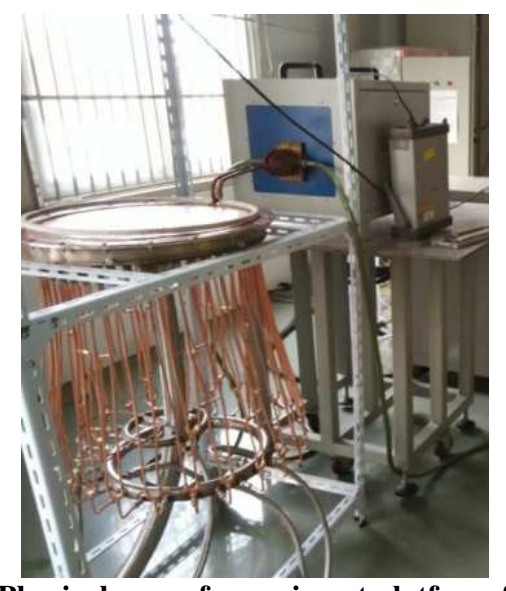

Figure 3: Physical map of experiment platform for cooling technology of high heating target

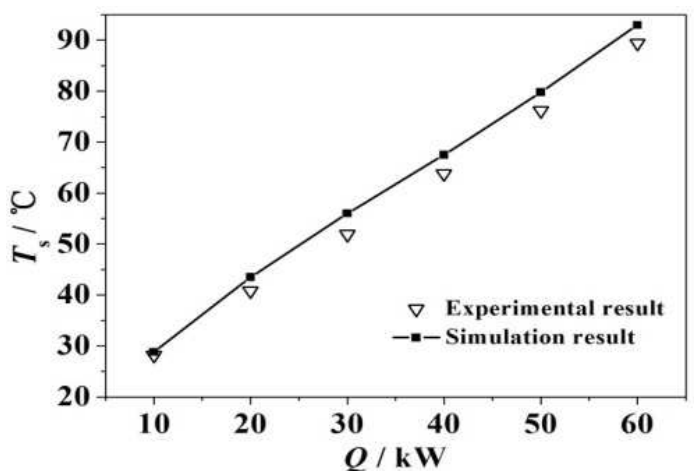

Figure 4: comparison of experimental and numerical simulation results

\section{RESULTS}

In this paper, the design and progress of HINEG project is presented. HINEG-I has both steady and pulse beams. The intensity of the steady beam is up to $10^{12} \mathrm{n} / \mathrm{s}$. The FWHM of pulse beam will less than $1.5 \mathrm{~ns}$. The installation of HINEG-I has been finished and is commissioning in December, 2015. HINEG-II is a steady D-T fusion generator. The intensity is $10^{14-15} \mathrm{n} / \mathrm{s}$. In order to achieve so high intensity, multi-accelerators array and high heating load rotating tritium target will be used.

HINEG-I and HINEG-II could be used in the areas of fusion nuclear data and fusion neutron transport in complicate medium, irradiation damage and activation test of fusion material, and so on. HINEG can also be utilized for radiation protection. For example, in biological research field, HINEG can be applied to 
neutron irradiation breeding, neutron cancer treatment, biological protection, etc... Recently, the DNA lesions and cell transformation which were induced by neutron irradiation were test, and further the chromosome aberration, tumorigenesis were examined in animal experiments to explore how the neutron irradiation act on living body and its mechanisms. The results can contribute on developing new antitumor treatment, and further research will be sustained carried on HINEG.

\section{Acknowledgment}

This work is supported by the Strategic Priority Science \& Technology Program of the Chinese Academy of Sciences (NO. XDA03040000) and ITER 973 Program (No. 2014GB112001). We would further like to thank the great help from the other members of FDS Team in this research.

\section{References}

1) A.Serikov, U.Fischer, C.S.Pitcher, A.Suarez, and B.Weinhorst, Computational Challenges for Fusion Neutronics for ITER Ports. SNA+MC 2013, Paris, France, (2013).

2) http://www-nds.iaea.org.

3) R.A. Forrest, "Nuclear Science and Data Need for Advanced Nuclear Systems," Energy Procedia, 7, 540-552, (2011).

4) TANG Jing-yu, JING Han-tao, XIA Hai-hong et al., "Key Nuclear Data Measurements for advanced Fission Energy and White Neutron Source at CSNS," Atomic Energy Science and Technology, 47, 1089-1095, (2013).

5) Y. Wu, J. Song, H. Zheng, et al., "CAD-Based Monte Carlo Program for Integrated Simulation of Nuclear System SuperMC," Ann. Nucl. Energy, 82: 161-168, ( 2015).

6) Q. Huang, FDS Team, "Development status of CLAM steel for fusion application," J. Nucl. Matter. 455, 649-654, (2014). 